\title{
Development of a radiographic dental implant guide for identification of dental implant types
}

SADJ September 2020, Vol. 75 No. 8 p432 - p437

L Vermeulen', A Speelman², V Daries ${ }^{3}$, V Philips ${ }^{4}$

\begin{abstract}
Introduction

Identification of dental implant types can be a complex process for inexperienced health care professionals. Dental implants can have subtle differences in their morphology, which make it difficult to distinguish them from one another.
\end{abstract}

The unique appearance of dental anatomy and the placement of custom restorations ensure accurate identification of bodies or human remains when radiographic techniques are correctly applied.

\section{Aims and objectives:}

To develop a radiographic dental implant guide for ten common dental implant types currently used in the Western Cape, South Africa; using their morphological characteristics observed on pantomographs.

\section{Design}

The methodology considered for this research study was a positivist approach through a quantitative, exploratory, non-experimental research design.

\section{Author affiliations:}

1. Lisa Vermeulen: MSc (Diagnostic Radiography), Cape Peninsula University of Technology, Cape Town, South Africa. The principal author is younger than 35 years of age.

2. Aladdin Speelman: MAppSc Medical Imaging (Computerised Tomography), Head of Department: Medical Imaging and Therapeutic Sciences, Faculty of Health and Wellness Sciences, Cape Peninsula University of Technology, Cape Town, South Africa. ORCID Number: 0000-0001-9183-6476

3. Valdiela Daries: Masters in Public Health, Lecturer: Department: Medical Imaging and Therapeutic Sciences, Faculty of Health and Wellness Sciences, Cape Peninsula University of Technology.

4. Vincent Philips: BDS; MChD, FC Path S.A. (Oral pathology), Dip Maxillo-Facial Radiology, PhD, DSc., Professor: Oral and MaxilloFacial Department, University of the Western Cape, Cape Town, South Africa.

Corresponding author: Lisa Vermeulen

Cape Peninsula University of Technology, Cape Town, South Africa.

Email: vermeulenlisa93@gmail.com

Author contributions:

Lisa Vermeulen: Principal author - 60\%

Aladdin Speelman: Co-author - $15 \%$

3. Valdiela Daries: Co-author $-15 \%$

4. Vincent Philips: Co-author $-10 \%$

\section{Methods}

Ten commonly used dental implants were radiographed at straight tube (ST), off-centre (OC) and severe offcentre (SOC) angles to create a reference instrument.

Two reviewers used the morphologies of the different dental implant types, namely the apex, thread and neck, observed on ante-mortem pantomographs, and compared it to the appearance of the dental implants in the reference instrument to make a positive identification match. The straight tube image of all ten dental implant types in the reference instrument was used as the initial point of reference to positively identify the morphological characteristics of each dental implant type on the pantomographs.

\section{Results}

A total of 380 dental implants could be identified on 105 pantomographs reviewed. Of the 380 dental implants, 350 dental implants (91\%) were identified as dental implant types listed in the reference instrument while 30 dental implants were identified as another type of dental implant type not listed in the reference instrument.

A total of 208 dental implants (54.2\%) could be positively identified on the ante-mortem pantomographs using the straight tube images in the reference instrument. The morphological characteristics of the dental implant types were described using $\mathrm{x}$-ray imaging of dental implants. The ten commonly used dental implants types could be positively identified by two independent reviewers and based on this a radiographic dental implant guide was developed.

\section{Conclusion}

Each dental implant type had unique morphological characteristics as well as similarities which enabled distinction between the different dental implant types.

The dental implant guide developed could be used by dentistry and radiography students. The dental implant guide may be useful in the field of forensic dentistry and forensic radiology. 


\section{INTRODUCTION}

Implantology has become more popular, accessible and of great value globally, therefore more health care professionals need to appreciate its application to identify different types of dental implants clinically and in forensic dentistry. Clinical and radiographic records of dental implant procedures are becoming widely and increasingly available and used during forensic identification of human remains. ${ }^{1}$

Forensic dentistry plays a key role in identifying human remains that cannot be identified visually or by other means; these remains include the victims of violent crime, fires (charred bodies), motor vehicle accidents and accidents on duty. Studies have shown that in cases of single or multiple deaths, scientific identification of human remains utilising forensic dentistry, is often the most successful source of identification. ${ }^{2,3}$ Dental identification of human remains consists of a very complex procedure that makes it necessary during the investigation process to use and compare unique dental identifiers. ${ }^{1}$

The different types of dental implants vary in morphology and in conjunction with the unique appearance of dental anatomy, and the placement of custom restorations such as dental implants, has been found to accurately assist in the identification of human remains. ${ }^{1}$ Dental implants have unique and overlapping morphological characteristics which for the untrained eye, are difficult to distinguish. The purpose of this study was to develop a dental implant guide using the unique morphological characteristics of the ten most common dental implant types used in the Western Cape, South Africa.

\section{METHODS}

The methodology applied during this research study was a positivist approach through a quantitative, exploratory, non-experimental research design. Ten dental implant types (Table 1) were radiographically imaged under nonclinical conditions.

\begin{tabular}{|c|c|c|}
\hline & Type of dental implant & Specifications \\
\hline 1. & Bicon & Example dental implant \\
\hline 2. & Biomet & Full Osseotite $3.25 \mathrm{~mm} \times 11.5 \mathrm{~mm}$ \\
\hline 3. & Champion & Example dental implant \\
\hline 4. & Megagen & AnyRidge $4 \mathrm{~mm} \times 10 \mathrm{~mm}$ \\
\hline 5. & MIS & MIS7 internal hex $6 \mathrm{~mm} \times 10 \mathrm{~mm}$ \\
\hline 6. & Neodent & Example dental implant \\
\hline 7. & Nobel Biocare & NobelActive $®$ \\
\hline 8. & Southern & IB $3.75 \mathrm{~mm} \times 12 \mathrm{~mm}$ \\
\hline 9. & Straumann & Example dental implants ( 3 ) \\
\hline 10. & Zimmer & SwissPlus (2) \\
\hline
\end{tabular}

Prior to the individual imaging of the ten dental implant types, the name of each dental implant type was registered on Carestream (software) on an Asus Pro Windows: laptop with i7 processor that was connected to a digital detector with dimensions: $27.6 \mathrm{~mm} \times 37.7 \mathrm{~mm}$; resolution: $24 \mathrm{lp} / \mathrm{mm}$.

Before the dental implant was placed on the digital detector, the name of the dental implant type (Table 1) was selected on the laptop computer. This ensured that the dental implant being radiographed corresponded with the dental implant type selected on the computer program in order to correctly label the dental implant on the computer system.

The exposure was set at 70kV (dental x-ray units usually operate between $50 \mathrm{kV}$ and $90 \mathrm{kV}$ ), $8 \mathrm{~mA}$ and $0.4 \mathrm{~s}$ (3.2mAs). Dental implants were removed from the plastic enclosure and were individually placed flat on the clean digital detector to be radiographed in 3 positions: straight tube (ST) which was positioned perpendicular to the dental implant, off centre (OC), a 5-degree central ray angulation, and severe off centre (SOC), a 30degree central ray angulation in the opposite direction (Figure 1A-C).

This method of exposing the dental implants 3 times was to create images of the dental implants that will correspond with the dental implants on the pantomographs. Dental implants on pantomographs may appear in an
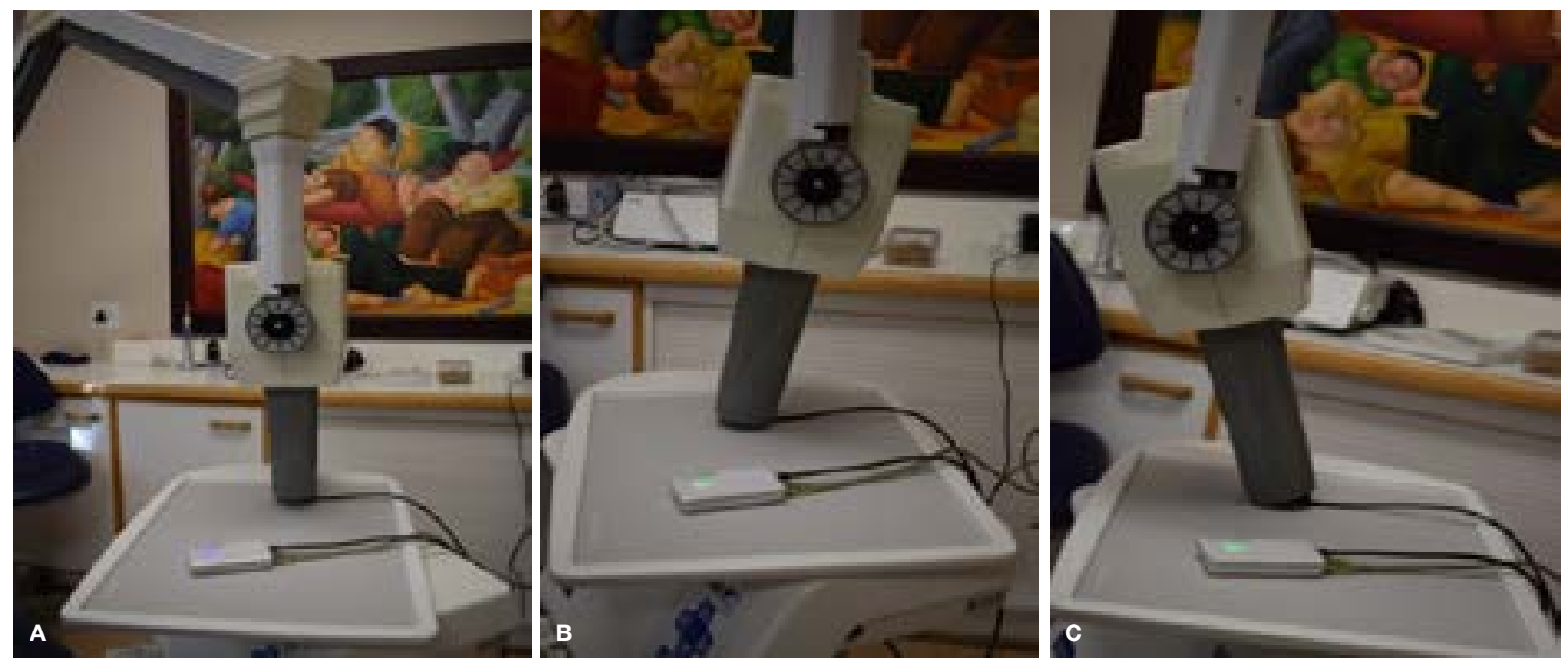

Figure 1A-C. Dental implant imaging representation: A - Straight tube (ST), B - Off centre (OC) 5-degree angulation, C - Severe off centre (SOC) 30-degree angulation in opposite direction. 
off centre (or oblique) position due to the position of the dental implant in the occlusion, or due to technique errors during actual radiographic acquisition of the pantomographs.

A total number of 36 images were taken and saved. Afterwards all the images were stored on a personal computer [Mecer Xpression, Model: W251HP, HDMI (High-Definition Multimedia Interface)], and saved in a folder named "Dental implant data".

All 36 images were backed up on a personal external hard drive, saved with the corresponding folder name "Dental implant data". Subsequently, a reference instrument was compiled by using the radiographs of the ten dental implants imaged at ST, OC and SOC angles.

Ethical approval to conduct this research study was obtained from the Research Ethics Committee of the Faculty of Health and Wellness Sciences, Cape Peninsula University of Technology as well as the Dean of the Faculty of Dentistry, University of the Western Cape. The ethical principles of the Declaration of Helsinki ${ }^{4}$ were upheld during this research study.

A total of 223 pantomographs, presenting with dental implants, were retrieved from the computer monitor of the Panorex $x$-ray unit at the research site. Of the 223 pantomographs, 105 were regarded as suitable for analysis.

Two reviewers (the researcher as radiographer and consultant periodontist) reviewed the dental implants present on the pantomographs; the review process was done independently. Each dental implant on the pantomographs was reviewed using morphological characteristics, namely the shape of the neck, appearance of the thread, and shape of the apex as seen on the reference instrument. Each of the dental implants was also independently compared with the OC image (5-degree central ray angulation) and SOC image (30-degree central ray angulation in opposite direction).

The researcher correlated with a specialist in the field in the few cases where the type of dental implants could not be positively identified. This verification was done after both reviewers had independently reviewed the dental implants on the pantomographs, using the reference instrument.

\section{RESULTS}

A total of 384 dental implants were in-situ on the 105 pantomographs analysed. Only nine of the ten different dental implant types radiographed, to create the reference instrument (listed in Table 1), were observed on the pantomographs.

An additional 5 dental implant types were identified on the pantomographs that were not included as part of the reference instrument, while 4 dental implants seen on the pantomographs could not be identified. In the sample of 384 dental implants, 380 dental implants in total were positively identified on the pantomographs as a specific dental implant type.

Frequency analysis was used to analyse the data for this research study. The Cohen's Kappa test was performed in Microsoft Excel to determine inter-observer reliability and to measure the agreement between the two reviewers. The researcher and the consultant periodontist both identified the dental implant types independently to ensure a non-bias and valid outcome of the test. The inter-observer agreement was $86.4 \%$ (Table 2).

\begin{tabular}{|c|c|}
\hline Number of dental implants viewed & 384 \\
\hline Number of dental implants identified: & 380 \\
\hline - Observer 1 (radiographer) & 332 \\
\hline - Observer 2 (consultant periodontist) & 380 \\
\hline Cohen's Kappa test \% & $332 / 384=86.4 \%$ \\
\hline
\end{tabular}

Of the 380 dental implants, 350 dental implants were identified as a dental implant type used for the reference instrument. A total of 208 dental implants (54.2\%) from nine dental implant types were identified using corresponding morphological characteristics (i.e. apex and/ or thread and/or neck) from the ST image (0-degree central ray angulation) used in the reference instrument. Each of the 208 dental implants was individually compared with the OC image (5-degree central ray angulation) and SOC image (30-degree central ray angulation in opposite direction) as well.

\begin{tabular}{|c|c|c|c|}
\hline Type of dental implant & $\begin{array}{l}\text { No. of implants identified from the } \\
\text { reference instrument using corresponding } \\
\text { morphological characteristics from the } \\
\text { Straight tube (ST) image }\end{array}$ & $\begin{array}{l}\text { No. of implants identified from the Off } \\
\text { Centre }(O C) \text { image }\end{array}$ & $\begin{array}{l}\text { No. of implants identified from the Severe } \\
\text { off centre (SOC) image }\end{array}$ \\
\hline Bicon & 13 & 13 & 13 \\
\hline Biomett & 12 & 12 & 2 \\
\hline Champion & 7 & 7 & 7 \\
\hline Megagen* & 0 & 0 & 0 \\
\hline MIS & 5 & 5 & 5 \\
\hline Neodent & 15 & 11 & 0 \\
\hline NobelActive & 56 & 56 & 56 \\
\hline Southern & 38 & 38 & 38 \\
\hline Straumann & 42 & 42 & 42 \\
\hline Zimmer & 20 & 20 & 9 \\
\hline Totals: & 208 & 204 & 172 \\
\hline
\end{tabular}


Of the 208 positively identified dental implants using the ST image (used as the point of reference image) in the reference instrument, 204 could be identified from the OC image, and 172 from the SOC image.

The OC and SOC images assisted the reviewers in confirming a positive identification of dental implant types. Unfortunately, four and 36 dental implants could not be

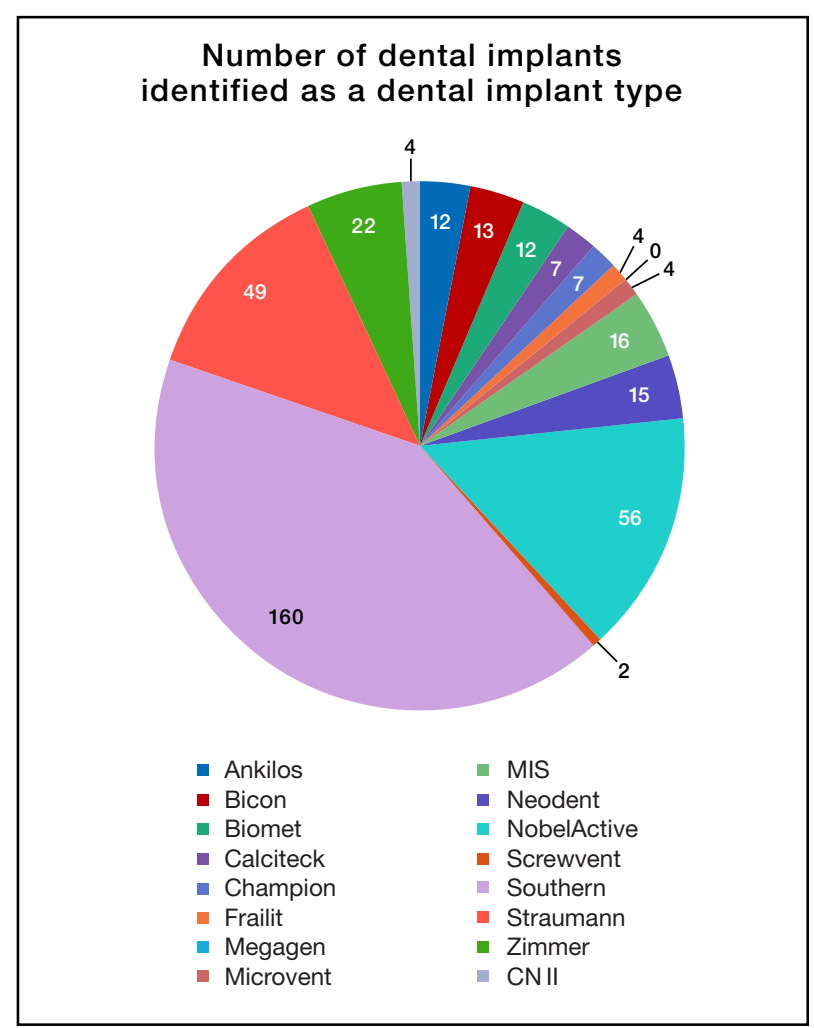

Figure 2. The number of dental implants identified from each dental implant type. identified from the $\mathrm{OC}$ and SOC images respectively. This indicates that the possibility of not identifying a dental implant type (owing to the unavailability of an ST image) may occur.

A total of 142 dental implants were identified as a dental implant type listed in the reference instrument using different variations of three morphological characteristics (apex, thread and neck), and in some cases the abutment was used for identification. These variations were observed by the researcher during the viewing of the pantomographs, and were confirmed by the consultant periodontist as additional information, to aid in the process of positively identifying dental implant types.

A total of 30 dental implants were identified as dental implant types not listed in the reference instrument. After a variation of morphological characteristics was used to analyse the unidentified dental implants, an additional $37 \%$ of dental implants were identified by the consultant periodontist as a dental implant type found in the reference instrument.

\section{DISCUSSION}

Dental implants are widely used to identify human remains by radiographic image recognition and geographic evaluation. If a human body is found and no dental records are available, it can be identified by the radiographic images of the dental implants.

The radiographs of dental implants are used to identify the manufacturers and different types of dental implants. Radiographs of dental implants are therefore useful for the odontologist to identify the victims. ${ }^{5}$

\section{Bicon}

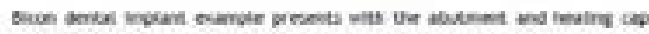
alicadr attachot to the ned of the inglant.

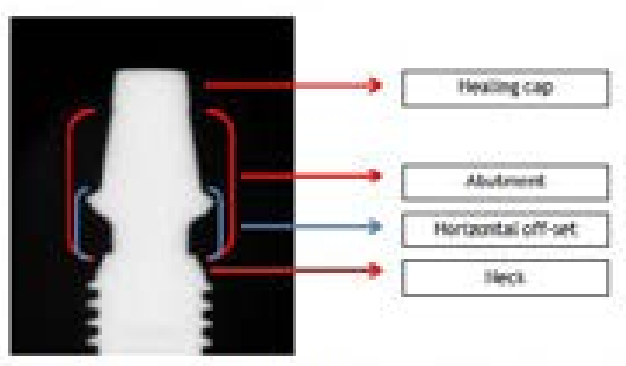

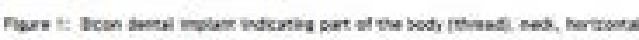

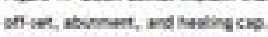

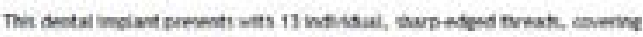
the body of the denes inpart in horiantas tivite. The apex of the dectes

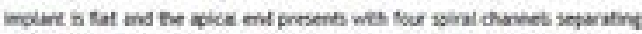

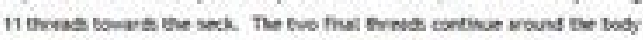

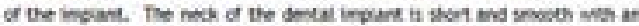

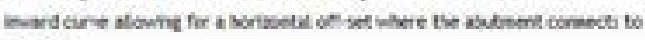
une now.
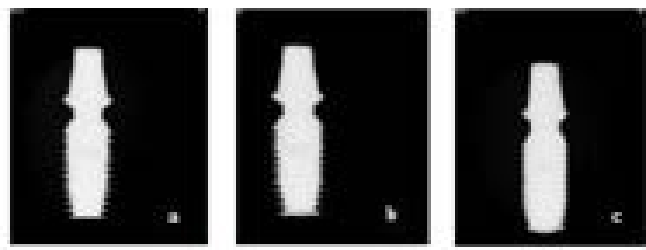

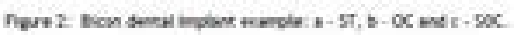

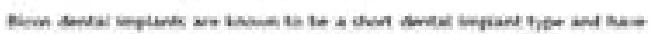

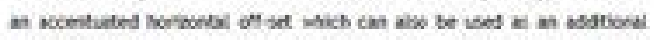

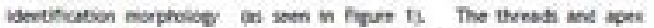

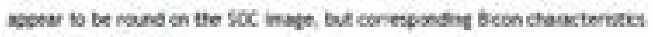
cinsalibe cierved

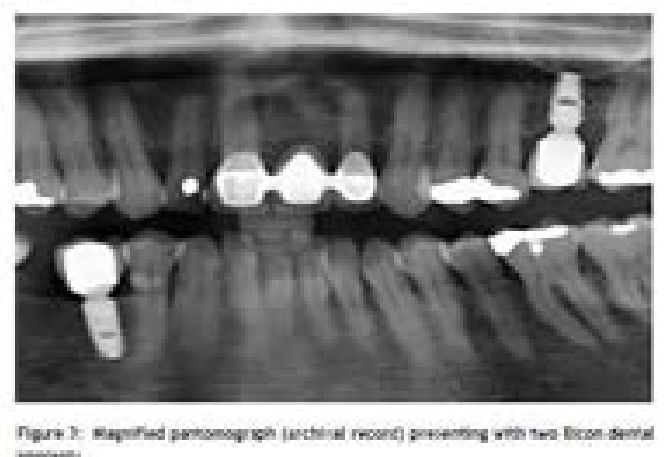

Figure 3. An excerpt from the radiographic dental implant guide presenting the Bicon dental implant. 
The identification of dental implant types remains challenging for inexperienced health care professionals and students. It can be argued that more learning mate rial needs to be made available that can assist with the identification of dental implant types on radiographs. The use of the morphology of different types of dental implants to identify various dental implant systems and human remains is still an unexplored field in South African forensic dentistry.

One of the main objectives of this research study was to describe the radiographic appearance of dental implants based on the morphological characteristics, including the apex, thread and neck of each dental implant type observed. This description was based on the morphology of dental implants and whether they could be positively identified on pantomographs and allowed the researcher to develop a radiographic dental implant guide for the ten dental implant types (Figure 3).

Three publications s,6,7 $^{1,6}$ described the use of dental implants during the identification of human remains. The literature indicated that in all three cases dental implants within the occlusion were successfully used to make a positive identification of human remains.

The type of dental implant used in the occlusion can be identified through morphological characteristics such as the connection, length, and diameter of the implant. Radiographic imaging is part of this process when comparisons need to be made between post-mortem and ante mortem images. ${ }^{8,9}$ Morphological features of dental implants depicted on radiographs may be used to develop a dental profile of the individual, and this can narrow the search to a smaller number of individuals, or eliminate certain candidates by taking into account the dental system employed. ${ }^{10}$

Dental implants, considering their morphological characteristics that differentiate between different types, give a supplementary layer of evidence during odontological identification, increasing the chances for a positive proof of identity. ${ }^{11}$ However, it is important that care should be exercised when using dental treatment radiographs for direct comparison against post-mortem radiographs as there are distortion and angulation factors that need to be considered.

This is a slight disadvantage, because if these factors are not considered, a positive identification might not be made. ${ }^{3}$ In comparison with a study done by Australian researchers ${ }^{12}$ where $51.3 \%$ of dental implants were identified, $54.2 \%$ of dental implants were identified during this research study by using the three morphological characteristics: i.e. apex, thread, and neck.

Previous research studies $2,8,10,13,14$ have indicated that dental implant types can be identified by their unique morphological characteristics. The ST image was used as the point of reference to identify dental implant types, with each positively identified dental implant type compared to the $\mathrm{OC}$ and SOC images thereafter.

In cases where there is distortion of dental implants, or where angulation of dental implants may occur owing to placement in the patient's dentition, it might be necessary to use the OC and/or SOC image to assist in making a positive identification of such dental implant types.

It was possible to develop a radiographic dental implant guide using dental implant types commonly used in the Western Cape, South Africa. During this research study, the reference instrument was compiled by acquiring radiographs of the referenced dental implants at ST, OC and SOC angles. The morphological characteristics of the shape, size and structure of the apex, thread and neck of each dental implant type were identified and used to differentiate between dental implant types; the images of the reference instrument were used and the radiographic dental implant guide was created.

A digital version of the dental implant guide was created (available on request from the principal author), and may be used for the education and training of radiography, dental and medical students to enhance their learning in identifying dental implant types on pantomographs. It is postulated that the dental implant guide may be used as an academic and clinical reference tool. The digital guide may also serve as a user friendly and easy to access guide for identifying different dental implant types in living persons or in the deceased.

The authors are of the opinion that the morphologies of dental implants play an important role during the identification process of unidentified persons. Identification of dental implant types can be a complex process for inexperienced health care professionals.

Dental implants can have subtle differences in their morphology, which make it difficult to distinguish them from one another. Dental records play an important role particularly in the identification of human remains, and this supplementary dental implant guide may support the identification process of unidentified human remains in South Africa.

\section{Limitations}

This research study only described the morphological characteristics of one (or in some cases two) dental implants from ten different dental implant types, which was a limitation. Future research studies should involve more dental implant types based on a broader geographical area, unlike this research study that only used dental implant types predominantly used in the Western Cape, South Africa.

The modern and improved technology of dental implant manufacturing companies has led to an increased variety of dental implant types which necessitates use of more varieties. This research study was conducted from a forensic perspective combining the identification of dental implant types as well as the use of dental records in human remains identification.

Very few publications were found using the morphological characteristics of dental implants for human identification purposes, which influenced contextualising the findings. It is recommended that more studies on this topic be conducted. 


\section{CONCLUSION}

Human remains can be successfully identified if dental implants are present. During any type of investigation where human remains are unidentifiable, forensic dentistry can be a very useful adjunct. Dental implants are still relatively new in the field of dentistry, and as it will become more commonly used, it will necessitate the use of morphological characteristics to identify different types of dental implants, as well as the possibility to identify human remains.

Each dental implant type has unique morphological characteristics as well as similarities which enable differentiation between the different dental implant types. It is important to examine all three characteristics namely the apex, thread, and neck in order to make a positive identification of the dental implant type.

\section{Acknowledgements}

The authors would like to thank Dr P. Wolfaardt for providing the sample dental implants and availing his practice for the imaging of the dental implant types. Also thank you to Dr R. Vermeulen for his contribution to the final manuscript.

\section{References}

1. Silva R.F, Franco A, Gratão de Castro M, Dumont J.A.V, Garcia R.R, Batista de Souza J. Dental Human Identification using Radiographic Records of Oral Implant Placement - a Case Report. Austin Journal of Forensic Science and Criminology. 2014: 1(1): 1-3.

2. Pretty A, Sweet D. A look at forensic dentistry. British Dental Journal. 2001; 190: 359.

3. Verma A.K, Kumar S, Rathore S, Pandey A. Role of dental expert in forensic odontology. National Journal of Maxillofacial Surgery. 2014: 5(1): 2-5.

4. World Medical Association (WMA). WMA Declaration of Helsinki - Ethical principles for medical research involving human subjects. 2018. https://www.wma.net/policies-post/ wma-declaration-of-helsinki-ethical-principles-for-medical-research-involving-human-subjects.

5. Nuzzolese E, Lusito S, Solarino B, Di Vella G. Radiographic dental implants recognition for geographic evaluation in human identification. Journal of Forensic Odontostomatol. 2008: 27: 8-11.

6. De Angelis D, Cattaneo C. Implant Bone Integration Importance in Forensic Identification. Journal of Forensic Sciences. 2015: 60(2): 505-8.

7. Berketa J.W, James H, Langlois N.E.I, Richards L.C. A study of osseo-integrated dental implants following cremation. Australian Dental Journal. 2014: 59(2): 149.

8. Brown R.M, Devenport J.S. Forensic Science: Advanced Investigations. Boston: Cengage Learning. 2012.

9. Berketa J.W, Hirsch R.S, Higgins D, James H. Radiographic Recognition of Dental Implants as an Aid to Identifying the Deceased. Journal of Forensic Sciences. 2010; b:55(1): 66.

10. Byraki A, Costea A.V, Curca G.C, Hostiuc S. Morphological analysis of dental implants - forensic significance. Romanian Journal of Legal Medicine. 2010: 18(3): 207-2012.

11. Dumancic J, Kaic Z, Njemirovskij V, Brkic H, Zecevic D. Dental identification after two mass disasters in Croatia. Croatia Medical Journal. 2001: 42: 657-62.

12. Soukoulis, S. Forensic odontology and a dental implant registry. 2016. https://www.linkedin.com/pulse/forensic-odontology-dental-implant-registry-dr-steven-soukoulis?trk=portfolio_article-card_title.
13. Michelinakis G, Sharrock A, Barclay C.W. Identification of dental implants through the use of Implant Recognition Software (IRS). International Dental Journal. 2006: 56(4): 203-8.

14. Berketa J, James H, Marino V. Survival of batch numbers within dental implants following incineration as an aid to identification. Journal of Forensic Odonto-Stomatology. 2010; a:28(1): 1-4. 